\title{
A case report of transmission and disease caused by Mycobacterium caprae and Mycobacterium bovis in Lima, Peru
}

\author{
Amber Shrestha', Janeth Picoy ${ }^{2}$, Arturo Torres ${ }^{3}$, David A. Moore ${ }^{4}$, Robert H. Gilman ${ }^{5,6}$, Jorge Coronel ${ }^{7}$ and \\ Louis Grandjean ${ }^{1,8^{*}}$
}

\begin{abstract}
Background: The Tuberculosis (TB) burden in Peru is significant with respect to both disease morbidity and mortality. Furthermore the recent diversification of farming enterprise to include a wide range of animal species has necessitated the consideration of members of the Mycobacterium Tuberculosis Complex (MTBC) with the potential for zoonotic transmission. M. bovis and M. caprae, a lesser known member of the MTBC exhibit an exceptionally wide host spectrum in animals and are capable of causing disease in humans. M. bovis has a predictable resistance profile which includes resistance to pyrazinamide. Thus, failure to identify $M$. bovis as the causative agent in reported TB cases leads to higher levels of treatment failure and contributes to the transmission of drug-resistant TB.
\end{abstract}

Case presentation: Reported here are the clinical presentations, investigations and treatment histories of two patients identified from a population level genotyping study in Lima, Peru that were at the time of treatment thought to be $M$. tuberculosis patients but in retrospect were spectated using whole genome sequencing as $M$. caprae and $M$. Bovis.

Conclusions: The cases reported here constitute convincing evidence that $M$. caprae and $M$. bovis are causative agents of TB infection in humans in Peru and underscore the importance of species-level MTBC member identification to effectively control and treat zoonotic TB. Furthermore these cases highlight the challenges of using clinical risk factors to identify cases of zoonotic TB in humans as their clinical presentation and transmission history is often difficult to distinguish from anthroponotic TB.

Keywords: Mycobacterium tuberculosis, Zoonotic disease, Caprae, Bovis, Case report

\section{Background}

In 2018 Tuberculosis (TB) claimed 1.5 million lives and was responsible for more deaths annually than HIV and Malaria combined making it the world's most deadly infectious disease [1]. Peru was the second biggest contributor to the TB disease burden in the World Health Organisation (WHO) region of the Americas, accounting

\footnotetext{
*Correspondence: I.grandjean@ucl.ac.uk

${ }^{1}$ Department of Infectious Disease, Great Ormond Street Hospital

for Children, London WC1N 3JH, UK

Full list of author information is available at the end of the article
}

for $13 \%$ of the 289,000 new and relapse TB cases reported in 2018 [1]. This translates to an annual incidence of 123 cases per 100,000. These figures remind us that TB is a significant threat to the population of Peru and underscores the importance of continued efforts to target and eradicate TB worldwide [1].

The aetiological agents of $\mathrm{TB}$ are members of the Mycobacterium Tuberculosis Complex (MTBC); namely M. tuberculosis, M. africanum, M. bovis, M. microti, M. canetti, M. caprae and M. pinnipedii [2]. The zoonotic potential of the MTBC is well established. This is perhaps 
best exemplified by $M$. bovis which exhibits an exceptionally wide host spectrum [2-4]. While M. bovis is classically associated with disease in cattle, it has also demonstrated its pathogenicity across a wide range of domestic and wild animals including but not limited to cats, dogs, badgers, bison, deer, goats and sheep [2]. Furthermore the zoonotic transmission of $M$. bovis is well documented and occurs primarily through the consumption of unpasteurised animal products contaminated with mycobacteria and through the inhalation of infectious aerosols during the close contact of humans with infected cattle. The respiratory route of human-tohuman contagion is thought to be less likely for $M$. bovis relative to $M$. tuberculosis but this is disputed [5-8].

$M$. bovis exhibits an intrinsic resistance to pyrazinamide, a first-line anti-TB agent that is included in the standard treatment regimen offered to new TB patients [8-10]. Furthermore, reports of primary resistance to isoniazid, rifampicin and streptomycin primary resistance have recently emerged [3, 8]. Multidrug resistant (MDR) forms of M. bovis have also been isolated [8].

The recent diversification of farming enterprise to include animal species other than cattle has necessitated the consideration of other aetiological agents of zoonotic TB including the opportunistic human pathogen, $M$. caprae. Figure 1 shows the phylogeny of $M$. caprae, $M$. bovis and their genetic distance from M. tuberculosis. In contrast to $M$. bovis, resistance of $M$. caprae against firstline anti-TB agents is a rarity [11]. Additionally $M$. caprae exhibits a more restricted host-range relative to $M$. bovis, its primary animal reservoir is small ruminants such as sheep and goat [12]. Finally M. caprae is not globally distributed and rarely occurs due to strain importation. The species is thought to be confined to Europe $[13,14]$.

Here we present the treatment history and clinical outcomes of two patients identified from a population level genotyping study in Peru that were at the time of treatment thought to be $M$. tuberculosis patients but in retrospect were speciated using whole genome sequencing as M. caprae and M. bovis.

\section{Methods}

\section{Data collection}

Sample collection was undertaken as per Grandjean et al., [15]. A total of 2139 patient with symptoms of TB presented to health posts in the regions of Callao and Lima South between December 2008 and January 2010. Sputum culture, microscopic observation drug susceptibility assay (MODS), serial smear microscopy, DNA extraction, Mycobacterial Interspersed Repetitive Units (MIRU) typing, Spoligotyping and whole genome sequencing were performed as previously described [15-18]. The dataset included three $M$. caprae isolates and one $M$. bovis isolate. Data pertaining to one M. bovis and one $\mathrm{M}$. caprae case was collected by chart review. The charts of the remaining $M$. bovis and $M$. caprae cases could not be located and as a result these cases were excluded from the current case review.

\section{Ethical approval}

Ethical approval was obtained from the Institutional Review Board of the Universidad Peruana Cayetano Heredia as part of a previously published population level study (IRB00001014) (Approval Number 57492) [15-17]. Institutional approval was obtained from the Peruvian Ministry of Health and the specific regional tuberculosis control programmes in the Callao and Lima South regions of Lima, Peru. Informed written consent was obtained from all study participants.

\section{Case presentation}

\section{Case 1. M. caprae}

In 2009, a 29-year-old Peruvian male presented with a 3-month history of a chronic cough productive of yellow/ green coloured sputum with occasional haemoptysis, associated with significant fatigue, diminished appetite, weight loss (7 kg in two months), night sweats and back pain.

A chest X-ray demonstrated right-sided apical cavitation with prominent bilateral hilar lymphadenopathy. A sputum specimen was positive for acid fast bacilli. Microscopic Observed Drug Susceptibility (MODS) testing did not indicate drug resistance.

The patient was treated with a 4-drug (rifampicin, isoniazid, pyrazinamide and ethambutol) anti-TB antibiotic regimen for a course of two months. Rifampicin and isoniazid was continued for a further four months. Sputum smears became negative after one month of treatment suggestive of a favourable disease progression and six subsequent sputum smear samples were negative. A repeat chest radiograph demonstrated right-sided apical fibrous reticular infiltrates consistent with treated inactive TB. The patient was considered to be in remission and remained asymptomatic thereafter.

The patient was born in the Callao region of Peru (population size 800,000). The patient shared one bedroom with his wife, son and parents in law. The patient's locality is known for a pig farm that employs many of the region's residents. Our patient's bother worked on this pig fam and had regular contact with our patient. 1-month prior to our patient's presentation the patient's brother was successfully treated for TB, the causative MTBC agent was not identified. The patient did not report any other contacts with domestic or wild animals and denies ingesting unpasteurised 


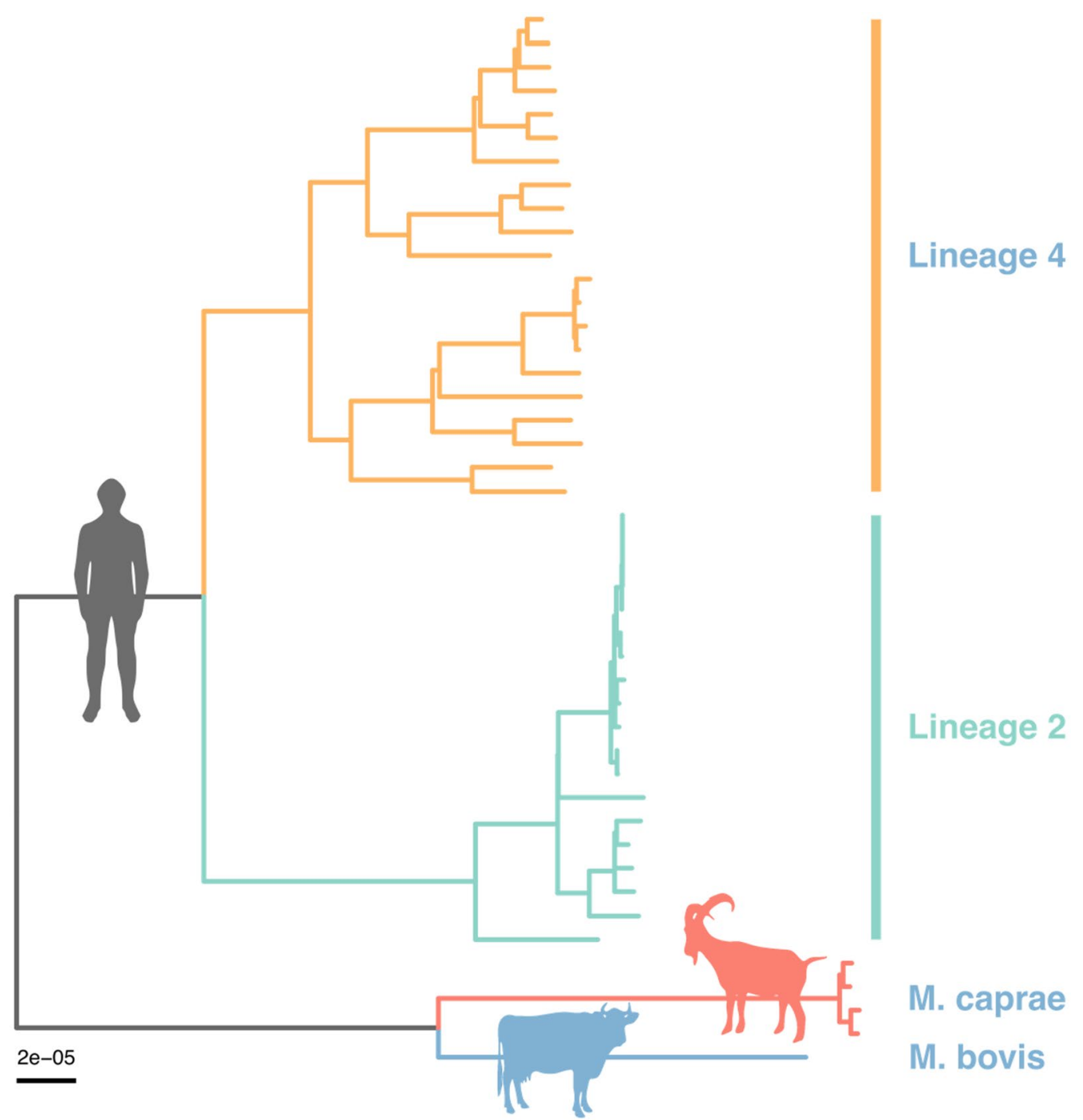

Fig. 1 Phylogeny of M. tuberculosis, M. bovis and M. caprae. Shown in the figure is the maximum likelihood tree of M. tuberculosis, M. bovis and M. caprae based on the single nucleotide polymorphism alignment from Illumina whole genome sequencing of 2,139 clinical isolates isolates in Lima Peru

dairy products. There was no further household transmission of TB between our patient and the other members of his household.

The patient's sputum sample was processed on both liquid (MODS) and solid Ogawa medium. An aliquot was sub-cultured and underwent Spoligotyping after DNA extraction at the Universidad Peruana Cayetano Heredia (Lime, Peru) $[15,19,20]$. The isolate was identified as $M$. caprae and was further genotyped using a 15-loci MIRU-VNTR analysis at the Kobe Institute (Kobe, Japan) following established protocols [21]. Subsequent whole genome sequencing identified the sample as M. caprae.

\section{Case 2. M. bovis}

In 2008, a 64-year-old Peruvian male presented with a 3-month history of a productive cough with haemoptysis and shortness of breath. A sputum smear was positive for acid fast bacilli. MODS testing did not indicate drug resistance. A chest radiograph demonstrated apical cavitation of the left upper and middle lung lobe with blunting of the left costo-diaphagmatic angle.

The patient was treated with the standard 4-drug regimen for a total course of 6 months. Sputum smears became negative after one month of treatment suggested favourable disease progression and five subsequent sputum smears were negative. 
However, 1 month after the patient stopped treatment he deteriorated clinically and had three positive smears. He was treated with a second line anti-TB regimen of ethambutol, pyrazinamide, ethionamide, ciprofloxacin, cycloserine, kanamycin and para-aminosalicylic acid for a total duration of 18 months. Despite initial improvements in symptoms, the patient relapsed again on 2nd line therapy and died of respiratory failure in 2012.

The patient lived alone in the region of Lima South (population size 1,200,000). Prior to his initial hospitalisation the patient had spent two months visiting family in the city of Huánuco in central Peru. This region has the greatest density of cattle farms and grazing cows in the country. While the consumption of unpasteurised milk in Huánuco is commonplace, our patient denies consuming unpasteurised dairy products.

The patient's sputum sample was processed on both liquid (MODS) and solid Ogawa medium. An aliquot was sub-cultured and underwent Spoligotyping after DNA extraction at the Universidad Peruana Cayetano Heredia (Lime, Peru) $[15,19,20]$. The isolate was identified as $M$. bovis and was further genotyped using a 15-loci MIRUVNTR analysis at the Kobe Institute (Kobe, Japan) following established protocols [21]. Subsequent whole genome sequencing identified the sample as $M$. bovis.

\section{Discussion and conclusion}

The cases reported here constitute convincing evidence that $M$. caprae and $M$. bovis are causative agents of TB infection in humans in Peru. Furthermore these cases highlight the challenges of using clinical risk factors to identify cases of zoonotic TB in humans as their clinical presentation and transmission history is often difficult to distinguish from anthroponotic TB.

In Peru small ruminant farming is a meaningful source of enterprise and sustenance by providing meat, milk and wool to Peruvian communities who's semi-arid and arid land often lacks the rich pasture necessary for cattle farming. More recently small ruminants such as the domestic caprid, the alpaca and the llama have demonstrated their ability to generate revenue in Peru's growing tourism industry and this has further bolstered their value. As the primary animal reservoir for $M$. caprae, it could be assumed that Peru's large inventory of small ruminants would result in a large proportion of reported TB cases being attributed to $M$. caprae. However $M$. caprae accounts for a mere $0.3 \%$ of human TB cases with the great majority of these cases being reported in Europe [14]. In fact, to the best of our knowledge there are only two documented cases of $M$. Caprae infection in humans outside of Europe; both described in Morocco [22]. Similarly, $M$. caprae is rarely isolated from animals outside of Europe. Isolates from cattle have been described in Tunisia and Algeria only, while the only documentation of $M$. caprae in China comes from Zeng et al., who described its isolation from one reindeer and one sheep [13, 23]. Thus, the case presented here represents the first documentation of zoonotic $M$. caprae infection in humans in The Americas, and only the fourth documented isolation outside of continental Europe.

It is unlikely that the apparent scarcity of $M$. caprae outside of Europe reflects the true epidemiology of zoonotic $M$. caprae in Peru but rather the lack of differentiation between $M$. caprae and the other members of the MTBC as the offending pathogen in reported TB cases $[11,13]$. M. caprae and M. tuberculosis have a very similar disease evolution and they are both typically drug-susceptible [14]. It is interesting to note that while $M$. caprae and $M$. bovis are often associated with extrapulmonary presentations of TB, both of our patients presented with a symptoms profile consistent with classical pulmonary TB $[13,14,24]$. Additionally when asked as part of a TB-specific history taking proforma included in each of the patients charts, neither of our patients reported consuming unpasteurised dairy products and both denied direct contact with cattle or caprine herds. This highlights the similarities in clinical picture between TB caused by $M$. bovis or M. caprae and TB infection caused by M. tuberculosis. Furthermore this would suggest that the screening questions addressing the risk factors of zoonotic TB, namely questions pertaining to animal product consumption and/or contact, are not as sensitive as one would have thought.

In developed countries 'whole herd test and slaughter' campaigns have significantly reduced but not eradicated the public health risk of zoonotic TB [25]. Herd eradication programmes aimed at reducing the zoonotic TB burden in Peru are scarce and are implemented on a regional basis relying on the active participation of farmers. These programmes have struggled to target the last vestiges of bovine TB which likely persist because of the failure of such programmes to include animal populations other than domestic cattle [25]. M. caprae's existence in caprine herds, which often coinhabit with cattle herds, represents a significant pool of zoonotic TB capable of causing disease in humans that is not targeted by herd eradication programmes. Describing the true disease burden of M. caprae in Peru would allow policy makers to decide whether caprine herds' contribution to the zoonotic TB pool in Peru warrants their inclusion in regional eradication programmes.

The rationale for the upfront species-level identification of $M$. bovis is clear. M. bovis has a predictable resistance profile which includes but is not limited pyrazinamide resistance. Thus, failure to identify $M$. bovis as the causative agent in reported TB cases has 
the potential to lead to higher levels of treatment failure and contributes to the transmission of drug-resistant TB. This was exemplified by our patient, Case 2. whose infection failed to respond to the first line drug regimen which included Pyrazinamide. In 2019 the WHO named Peru as one of the top 30 countries with the highest burden of MDR-TB [1]. M. Bovis is a known causative agent of MDR-TB and characterizing its epidemiology in Peru has important therapeutic implications.

Limitations of this study include the use of chart review as the primary method of data collection. It was not possible to contact our patients directly and this limited the quantity and quality of the data presented here. Furthermore, it was not possible to gather data from two of the M. caprae cases isolated as part of our parent study and this limits our ability to generalize the findings discussed here.

Recent large-scale genotyping studies have provided a population level view of circulating strains of TB in the region of Lima, Peru. As a result of this, the prevalence of human disease due to the different members of the MTBC has been quantified [16]. The case reports presented here underscore the importance of species-level MTBC member identification in efforts to control and effectively treat zoonotic TB caused by M. bovis and M. caprae.

\footnotetext{
Abbreviations

M. bovis: Mycobacterium tuberculosis; M. caprae: Mycobacterium caprae; $M$. Tuberculosis: Mycobacterium bovis; MTBC: Mycobacterium tuberculosis complex; TB: Tuberculosis; WHO: World Health Organisation; MDR-TB: Multi-drug resistant tuberculosis; 15 Locus MIRU-VNTR: 15-Locus Mycobacterial Interspersed Repetitive Unit Variable Number Tandem Repeat; MODS: Microscopic Observation Drug Susceptibility Assay.
}

\section{Acknowledgements}

Not applicable.

\section{Authors' contributions}

AS collected the patient data recorded in Case 1. M. caprae and Case 2 $\mathrm{M}$. bovis and contributed significantly to the write up of the following manuscript sections: Background, Methods, Results, Discussion, Conclusions. JP assisted with research question conceptualisation and patient identification. AT performed the Phylogeny of M. tuberculosis, M. bovis and M. caprae and significantly contributed to the write up of the discussion. DM assisted with research conceptualisation and patient identification. RG assisted with research conceptualisation and patient identification. JC assisted with research conceptualisation and patient identification. LG conceived the conceptual idea underpinning the research, assisted with the collection of patient data and significantly contributed to the write up of the following manuscript sections: Background, Results, Discussion, Conclusions. All authors read, edited and approved the final manuscript.

\section{Funding}

Not applicable.

\section{Availability of data and materials}

Figure 1. titled, Phylogeny of M. tuberculosis, M. bovis and M. caprae depicts the maximum likelihood tree of M. tuberculosis, M. bovis and $M$. caprae based on the single nucleotide polymorphism alignment from Illumina whole genome sequencing of 2,139 clinical isolates isolates in Lima Peru. This figure is derived from 2,139 clinical isolates collected as part of the following paper:

Grandjean L, Iwamoto T, Lithgow A, Gilman RH, Arikawa K, Nakanishi N, Martin L, Castillo E, Alarcon V, Coronel J, Solano W, Aminian M, Guezala C, Rastogi N, Couvin D, Sheen P, Zimic M, Moore DA. The Association between Mycobacterium Tuberculosis Genotype and Drug Resistance in Peru. PLoS One. 2015 May 18;10(5):e0126271. https://doi.org/10.1371/journal.pone.0126271. PMID: 25,984,723; PMCID: PMC4435908.

The original data set is available to request from the original authors of that paper.

\section{Declarations}

\section{Ethics approval and consent to participate}

Ethical approval was obtained from the Institutional Review Board of the Universidad Peruana Cayetano Heredia as part of a previously published population level study (IRB00001014) (Approval Number 57492)(15-17). Institutional approval was obtained from the Peruvian Ministry of Health and the specific regional tuberculosis control programmes in the Callao and Lima South regions of Lima, Peru.

\section{Consent for publication}

Informed written consent was obtained from all study participants in advance of their TB treatment and/or clinical outcomes including death.

\section{Competing interests}

There are no financial or non-financial competing interests.

\section{Author details}

'Department of Infectious Disease, Great Ormond Street Hospital for Children, London WC1N 3JH, UK. ${ }^{2}$ Department of Infectious Disease, Diresa Callao Jr, Colina \#879, Bellavista, 07016 Lima, Peru. ${ }^{3}$ Department of Infectious Disease, Imperial College London, South Kensington, London SW7 2AZ, UK. ${ }^{4}$ TB Centre, London School of Hygiene \& Tropical Medicine, London WC1E 7HT, UK. ${ }^{5}$ Laboratorio de Tuberculosis, Laboratorios de Investigación Y Desarrollo, Facultad de Ciencias Y Filosofía, Universidad Peruana Cayetano Heredia, Lima, Peru. ${ }^{6}$ Department of International Health, School of Public Health, Johns Hopkins University, Baltimore, MD, USA. 'Laboratorio de Investigación de Enfermedades Infecciosas, Universidad Peruana Cayetano Heredia: Lima, Lima, Peru.

${ }^{8}$ University College London, Gower St, Bloomsbury, London WC1E 6BT, UK.

Received: 30 September 2021 Accepted: 3 December 2021

Published online: 20 December 2021

\section{References}

1. Geneva: World Health Organization. WHO | Global tuberculosis report 2019. The WHO; 2019 [cited 2019 Nov 17]. Report No.: Licence: CC BY-NCSA 3.0 IGO. Available from: https://www.who.int/tb/publications/global_ report/en/

2. O'Reilly LM, Daborn CJ. The epidemiology of Mycobacterium bovis infections in animals and man: a review. Tuber Lung Dis. 1995;76:1-46.

3. Djemal SE, Camperio C, Armas F, Siala M, Smaoui S, Messadi-Akrout $F$, et al. Detection of a streptomycin-resistant Mycobacterium bovis strain through antitubercular drug susceptibility testing of Tunisian Mycobacterium tuberculosis complex isolates from cattle. BMC Vet Res. 2018;14(1):296.

4. Marianelli C, Amato B, Boniotti MB, Vitale M, Pruiti-Ciarello F, Pacciarini ML, et al. Genotype diversity and distribution of Mycobacterium bovis from livestock in a small, high-risk area in northeastern Sicily, Italy. PLoS Negl Trop Dis. 2019;13(7):e0007546.

5. Evans JT, Smith EG, Banerjee A, Smith RM, Dale J, Innes JA, et al. Cluster of human tuberculosis caused by Mycobacterium bovis : evidence for person-to-person transmission in the UK. Lancet. 2007;369(9569):1270-6.

6. Buss BF. Possible Airborne Person-to-Person Transmission of Mycobacterium bovis - Nebraska 2014-2015. MMWR Morb Mortal Wkly Rep. 2016;65. https://www.cdc.gov/mmwr/volumes/65/wr/mm6508a1.htm 
7. Guerrero A, Cobo J, Fortün J, Navas E, Quereda C, Asensio A, et al. Nosocomial transmission of Mycobacterium bovis resistant to 11 drugs in people with advanced HIV-1 infection. Lancet. 1997:350(9093):1738-42.

8. Bobadilla-del Valle M, Torres-González P, Cervera-Hernández ME, Martínez-Gamboa A, Crabtree-Ramirez B, Chávez-Mazari B, et al. Trends of Mycobacterium bovis isolation and first-line anti-tuberculosis drug susceptibility profile: a fifteen-year laboratory-based surveillance. PLoS Negl Trop Dis. 2015;9(9):e0004124.

9. de Jong BC, Onipede A, Pym AS, Gagneux S, Aga RS, DeRiemer K, et al. Does resistance to pyrazinamide accurately indicate the presence of Mycobacterium bovis? J Clin Microbiol. 2005;43(7):3530-2.

10. Patané JSL, Martins J, Castelão AB, Nishibe C, Montera L, Bigi F, et al. Patterns and processes of Mycobacterium bovis evolution revealed by phylogenomic analyses. Genome Biol Evol. 2017;9(3):521-35.

11. Kozińska M, Krajewska-Wędzina M, Augustynowicz-Kopeć E. Mycobacterium caprae - the first case of the human infection in Poland. Ann Agric Environ Med. 2019 [cited 2019 Nov 11]; http://www.aaem.pl/Mycobacter ium-caprae-the-first-case-of-the-human-infection-in-Poland,108442,0,2. html

12. Sykes JE. Canine and feline infectious diseases. St. Louis, Mo: Elsevier/ Saunders; 2014. $915 \mathrm{p}$.

13. Prodinger WM, Indra A, Koksalan OK, Kilicaslan Z, Richter E. Mycobacterium caprae infection in humans. Expert Rev Anti Infect Ther 2014;12(12):1501-13.

14. Nebreda T, Álvarez-Prida E, Blanco B, Remacha MA, Samper S, Jiménez MS. Peritoneal tuberculosis due to Mycobacterium caprae. IDCases. 2016:4:50-2.

15. Grandjean L, Iwamoto T, Lithgow A, Gilman RH, Arikawa K, Nakanishi N, et al. The association between mycobacterium tuberculosis genotype and drug resistance in Peru. PLoS ONE. 2015;10(5):e0126271.

16. Grandjean L, Gilman RH, Iwamoto T, Köser CU, Coronel J, Zimic M, et al. Convergent evolution and topologically disruptive polymorphisms among multidrug-resistant tuberculosis in Peru. PLOS ONE. 2017;12(12):e0189838.

17. Grandjean L, Gilman RH, Martin L, Soto E, Castro B, Lopez S, et al. Transmission of multidrug-resistant and drug-susceptible tuberculosis within households: a prospective cohort study. PLoS Med. 2015;12(6):e1001843.
18. Madrazo-Moya CF, Cancino-Muñoz I, Cuevas-Córdoba B, GonzálezCovarrubias V, Barbosa-Amezcua M, Soberón X, et al. Whole genomic sequencing as a tool for diagnosis of drug and multidrugresistance tuberculosis in an endemic region in Mexico. PLOS ONE. 2019;14(6):e0213046.

19. van Embden JD, Cave MD, Crawford JT, Dale JW, Eisenach KD, Gicquel $B$, et al. Strain identification of Mycobacterium tuberculosis by DNA fingerprinting: recommendations for a standardized methodology. J Clin Microbiol. 1993:31(2):406-9.

20. Ausubel FM. Short protocols in molecular biology: a compendium of methods from Current protocols in molecular biology. 5th ed. New York: Wiley; 2002. p. 2.

21. Supply P, Allix C, Lesjean S, Cardoso-Oelemann M, Rüsch-Gerdes S, Willery $E_{1}$ et al. Proposal for standardization of optimized mycobacterial interspersed repetitive unit-variable-number tandem repeat typing of Mycobacterium tuberculosis. J Clin Microbiol. 2006:44(12):4498-510.

22. Lahlou O, Millet J, Chaoui I, Sabouni R, Filali-Maltouf A, Akrim M, et al. The genotypic population structure of mycobacterium tuberculosis complex from moroccan patients reveals a predominance of Euro-American lineages. PLOS ONE. 2012;7(10):e47113.

23. Zeng $W$, Zhang $Y$, Zhao $X$, Huang $G$, Jiang $Y$, Dong $H$, et al. Occurrence of non-tuberculous mycobacteria species in livestock from northern China and first isolation of Mycobacterium caprae. Epidemiol Infect. 2013;141(7):1545-51.

24. Dürr S, Müller B, Alonso S, Hattendorf J, Laisse CJM, van Helden PD, et al. Differences in primary sites of infection between zoonotic and human tuberculosis: results from a worldwide systematic review. PLoS Negl Trop Dis. 2013;7(8):e2399.

25. Cousins DV. Mycobacterium bovis infection and control in domestic livestock: -EN-FR-ES-. Rev Sci Tech OIE. 2001;20(1):71-85.

\section{Publisher's Note}

Springer Nature remains neutral with regard to jurisdictional claims in published maps and institutional affiliations.
Ready to submit your research? Choose BMC and benefit from:

- fast, convenient online submission

- thorough peer review by experienced researchers in your field

- rapid publication on acceptance

- support for research data, including large and complex data types

- gold Open Access which fosters wider collaboration and increased citations

- maximum visibility for your research: over $100 \mathrm{M}$ website views per year

At $\mathrm{BMC}$, research is always in progress.

Learn more biomedcentral.com/submissions 